\title{
Analysis of Wave Normal and Poynting Vectors of the Chorus Emissions Observed by GEOTAIL
}

\author{
Isamu NAGANo ${ }^{1}$, Satoshi YAGITANI $^{1}$, Hirotsugu KoJIMA ${ }^{2}$, and Hiroshi MATSUMOTO ${ }^{2}$ \\ ${ }^{1}$ Department of Electrical and Computer Engineering, Kanazawa University, Kanazawa 920. Japan \\ ${ }^{2}$ Radio Atmospheric Science Center, Kyoto University, Uji, Kyoto 611, Japan
}

(Received December 28, 1994; Revised February 22, 1996; Accepted February 22, 1996)

\begin{abstract}
Many chorus emissions were observed in the frequency range from $200 \mathrm{~Hz}$ to $1 \mathrm{kHz}$ when the GEOTAIL spacecraft was skimming inside the dayside magnetopause. The Wave Form Capture (WFC) onboard GEOTAIL obtained simultaneous wave forms of two electric and three magnetic components of the emissions. Spectral structures of the chorus emissions observed by the WFC are roughly classified into four types: rising tone, falling tone, hook, and structureless. In this paper, as a preliminary report, the wave normal directions for each type of the emissions except for the structureless are derived by the Means' method around the geomagnetic field line to know their propagation characteristics. In addition, Poynting directions of the chorus emissions are derived by using their five electromagnetic components. Derived propagation directions of the chorus emissions along a GEOTAIL orbit indicate that they are generated around the dayside geomagnetic equator, which is consistent with the well-known model of chorus generation through cyclotron resonance in the dayside outer magnetosphere.
\end{abstract}

\section{Introduction}

Chorus emissions (VLF triggered emissions) in the Earth's and other planetary magnetospheres have been attracting many investigators because of their complicated but intriguing frequency-time behavior (a comprehensive review of chorus emissions is seen, for example, Sazhin and Hayakawa, 1992). Though various kinds of mechanisms have been proposed to explain the generation of nonlinear time evolution of narrowband rising or falling tones via interaction with energetic electrons (Omura et al., 1991), still there remains many uncertainties in generation/propagation mechanisms of the chorus emissions. One of the key factors significant to solve such a challenging problem should be a high-quality in-situ measurements of the frequency-time structures as well as propagation characteristics of the chorus emissions. In particular, wave normal and Poynting directions are very important to tell us exact source locations and to investigate generation/propagation mechanisms of the emissions in the magnetosphere.

So far several authors have made wave normal analysis for the magnetospheric chorus emissions observed by satellites. For example, the wave normal analysis for chorus emissions observed by OGO 5 showed that the emissions propagate at small angles (less than 20 degrees) to the geomagnetic field line around the geomagnetic equator (Burton and Holzer, 1974; Goldstein and Tsurutani, 1984), suggesting that the equatorial region is a possible source region of them. The off-equatorial wave normals are consistent with either a ducted or nonducted whistler mode propagation from the equatorial source region (Burton and Holzer, 1974). Also by using OGO 5 data, Cornilleu-Wehrin et al. (1976) found that wave normals vary inside each of chorus elements, and that falling tones take larger wave normal angles with respect to the geomagnetic field line than rising tones. The different wave normal directions for impulsive and rising types of the chorus emissions were derived by Hayakawa et al. (1984) for GEOS 2 observations.

Such in-situ measurements of chorus propagation characteristics have not only given evidence to the proposed generation/propagation mechanisms, but also suggested a new theoretical model for them. However, most of the previous analyses have not been based on the full six components of electromagnetic wave fields (usually only three magnetic components have been used to derive the wave normals). To 
discuss propagation characteristics of the chorus emissions more quantitatively, we need to examine Poynting flux in addition to the wave normal directions, using the full six electromagnetic components.

On the other hand, chorus emissions have been observed also by the GEOTAIL spacecraft in the dayside outer magnetosphere along its orbits skimming the magnetopause. The Plasma Wave Instrument (PWI) onboard GEOTAIL can be a very powerful tool to analyze the propagation characteristics of the chorus emissions, since it has the Wave Form Capture (WFC), which acquires two electric and three magnetic wave forms simultaneously (Matsumoto et al., 1994). By the WFC, both exact wave normal and Poynting directions as well as detailed frequency-time structures are obtained for the whistler mode waves like chorus emissions. In the present paper, as a preliminary report, we give a wave normal and Poynting analysis for the dayside chorus emissions observed by GEOTAIL whose frequency ranges are well covered by the WFC, to discuss their generation/propagation characteristics in the dayside outer magnetosphere.

In the next section we briefly describe the WFC and introduce a way to derive both wave normal and Poynting directions as a function of frequency and time on the basis of Maxwell's equations. Then in Section 3 propagation characteristics of the chorus emissions observed at the dayside magnetopause by GEOTAIL are analyzed by using five components of electromagnetic wave forms of the emissions. In Section 4 contents of the present paper are summarized.

\section{Analysis of Wave Form Data}

The WFC in the PWI subsystem acquires simultaneously five electromagnetic (two electric and three magnetic) wave forms between $10 \mathrm{~Hz}$ and $4 \mathrm{kHz}$. The observed wave form data are first stored in a $512-$ kByte onboard memory for $8.7 \mathrm{sec}$, and then slowly sent back to the ground during a time period of 275 sec. Thus we can get five electromagnetic continuous wave forms for $8.7 \mathrm{sec}$ every about $5 \mathrm{~min}$.

Frequency response of the electromagnetic sensors, two electric dipole antennas and a tri-axial magnetic search coil, and of an amplifier circuit in the PWI, should be considered to obtain absolute values of amplitudes as well as of phases of the five electromagnetic wave forms at the antenna sensors. Such an accurate calibration is important to derive exact wave normal and Poynting directions of the observed plasma waves. However, it was generally difficult to get the accurate frequency response of a dipole antenna because its impedance is strongly affected by a plasma sheath around it formed by the ambient plasma particles. To solve this problem, the PWI has a generator of a reference signal which is used to measure the impedances of the two types of the dipole antennas, WANT (Wire Antenna: a wire dipole antenna) and PANT (Probe Antenna: a pair of top-hat antennas with a sphere attached at each end of them) with a length of $100 \mathrm{~m}$ tip-to-tip (Matsumoto et al., 1994). In the calibration of wave forms of the observed chorus emissions at the dayside magnetopause, we include the antenna impedances of $325 \mathrm{pF}$ and $310 \mathrm{pT}$ for each monopole of WANT and PANT, respectively, which are actually measured by the PWI at the time of chorus observation (Tsutsui et al., 1996).

Each of the calibrated wave forms are then Fourier-transformed to give a frequency-time $(f-t)$ diagram, which is quite useful to examine fine $f-t$ structures of chorus emissions. For each element of the emissions seen in the $f-t$ diagram, we can derive wave normal and Poynting directions with a plane wave approximation as explained in the following.

We estimate wave normal vectors using a vector-product method (the Means' method (Means, 1972)). Here five components of electromagnetic fields, $E_{x}, E_{y}, B_{x}, B_{y}$, and $B_{z}$, are available by the WFC observation. For a plane wave field $\boldsymbol{E}$ or $\boldsymbol{B}$ oscillating with $\exp (j \omega t-\boldsymbol{k} \cdot \boldsymbol{r})$, from one of Maxwell's equations, $\nabla \cdot \boldsymbol{B}=0$, we have $\boldsymbol{k} \cdot \boldsymbol{B}=0$, where $\boldsymbol{k}$ is the wave normal vector. Then, the unit vector in the wave normal direction of a circularly or elliptically polarized wave (for example, a chorus emission), becomes

$$
\boldsymbol{u}_{k}=\frac{\boldsymbol{k}}{|\boldsymbol{k}|}=\operatorname{Im} \frac{\boldsymbol{B} \times \boldsymbol{B}^{*}}{\left|\boldsymbol{B} \times \boldsymbol{B}^{*}\right|},
$$


where "Im" and * means the imaginary part and the complex conjugate, respectively. In Eq. (1), $\boldsymbol{u}_{k}$ has ambiguity of \pm 180 degrees in its direction, which is determined by the estimation of the sign of the real part of the refractive index as described below.

From another Maxwell's equation, $\nabla \times \boldsymbol{E}=-\partial \boldsymbol{B} / \partial t$, we have $\boldsymbol{k} \times \boldsymbol{E}=\omega \boldsymbol{B}$, from which a refractive index of the wave is calculated as

$$
n=\frac{c B_{z}}{u_{k x} E_{y}-u_{k y} E_{x}} .
$$

The sign of the real part of the refractive index, $\operatorname{Re}(n)$, should be positive so that the correct unit vector in the wave normal direction is obtained here as

$$
\begin{cases}\boldsymbol{u}_{k} & \text { if } \operatorname{Re}(n)>0 \\ -\boldsymbol{u}_{k} & \text { if } \operatorname{Re}(n)<0\end{cases}
$$

which eliminates the \pm 180 -degree ambiguity for $\boldsymbol{u}_{k}$ in Eq. (1).

On the other hand, we can estimate the unobserved $E_{z}$ component from other five components, as

$$
E_{z}=-\frac{E_{x} B_{x}+E_{y} B_{y}}{B_{z}} .
$$

Thus Poynting flux of the wave is calculated from the full six components of electromagnetic fields,

$$
\boldsymbol{P}=\frac{1}{2} \operatorname{Re}\left(\boldsymbol{E} \times \frac{\boldsymbol{B}^{*}}{\mu_{0}}\right)
$$

where $\mu_{0}$ is the permeability in free space.

\section{Chorus Observations and Discussions}

Chorus emissions have been observed for almost all the GEOTAIL orbits skimming the dayside magnetopause. Figure 1 shows four examples of such orbits of GEOTAIL in the Geocentric Solar Magnetic (GSM) coordinate system, where the wave form data obtained by the WFC are available for some of the chorus emissions. In Fig. 1 the dotted curves represent these orbits of GEOTAIL, on each of which chorus emissions were observed along the solid portion. The numbers $1,2,3$ and 4 by the curves indicate the orbits on October 17-18 and November 4-5, 1992, April 29-30 and August 25, 1993, respectively. A predominance of chorus observations in the dawn-noon sector is consistent with the past satellite observations (for example, Tsurutani and Smith, 1977). Spectral structures of the chorus emissions observed by the WFC are roughly classified into four types: rising tone, falling tone, hook, and (banded-noise-like) structureless. Most of the observed emissions consisted of rising tones and structureless, with several hooks (including inverted hooks) and a few falling tones. The emission frequencies range from $200 \mathrm{~Hz}$ up to $1 \mathrm{kHz}$.

Figure 2 shows typical examples of the dynamic spectra for three emission types, rising tone, falling tone, and hook. In each example a magnetic field component for $8.7 \mathrm{sec}$ obtained by the WFC are represented as an $f-t$ diagram, where $1 \mathrm{pT} / \sqrt{\mathrm{Hz}}$ corresponds to $0 \mathrm{~dB}$.

Figure 2(a) shows several rising tones and a falling tone observed at 22:05UT on October 17, 1992. 

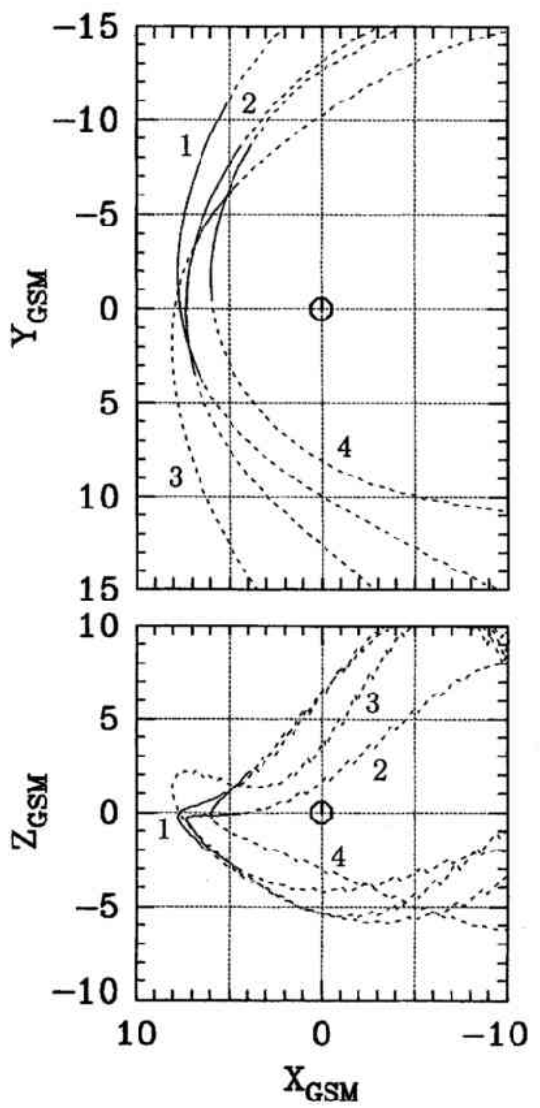

Fig. 1. Four dayside orbits of GEOTAIL in the GSM coordinate system. The numbers by the curves $1,2,3$ and 4 indicate the orbits on October 17-18 and November 4-5, 1992, and April 29-30 and August 25, 1993, respectively.

The falling tone appearing at $400 \mathrm{~Hz}$ at $4 \mathrm{sec}$ has an unusually long duration, the frequency going down to $220 \mathrm{~Hz}$ in about $4 \mathrm{sec}$, with a narrow band width less than $10 \mathrm{~Hz}$. Its intensity increases up to the maximum level of $41 \mathrm{pT}$ peak-to-peak at $6.3 \mathrm{sec}$, then gradually decreases down to one third of the maximum at $8 \mathrm{sec}$. This time variation of intensity may give us a clue to solve a generation mechanism of such a falling tone emission. The frequency range of the falling tone is between $0.15 \sim 0.3$ of $f_{H}$, where $f_{H}$ is the local electron cyclotron frequency observed by the Magnetic Field experiment (MGF) onboard GEOTAIL (Kokubun et al., 1994).

Figure 2(b) includes hook-type emissions observed around 23:39UT on November 4, 1992. The frequency of a strong emission observed first at $300 \mathrm{~Hz}$ at $4.5 \mathrm{sec}$ decreases down to $200 \mathrm{~Hz}$ in about 0.7 sec and then increases rapidly up to $370 \mathrm{~Hz}$. This frequency range corresponds to $0.1 \sim 0.15 f_{H}$.

Figure 2(c) shows several rising tones observed around 00:08UT on April 30, 1993. The center frequency of the rising tones is about $800 \mathrm{~Hz}$, which is about a quarter of the local $f_{H}$. The frequency variation for each rising tone, $d f i d t$, increases with time, from $0.5 \mathrm{kHz} / \mathrm{sec}$ to $1.0 \mathrm{kHz} / \mathrm{sec}$, while the amplitude increases from $200 \mathrm{pT}$ peak-to-peak up to $800 \mathrm{pT}$ peak-to-peak in the frequency range of $500 \mathrm{~Hz}$. This intensity is not extremely strong compared with the maximum intensity $(\sim 1040 \mathrm{pT})$ of chorus emissions observed by GEOS 2 (Parrot and Gaye, 1994).

The wave normal directions of the observed chorus emissions are calculated by Eq. (1) assuming a plane wave model, which has been considered valid for most of terrestrial chorus emissions (Goldstein and Tsurutani, 1984). 

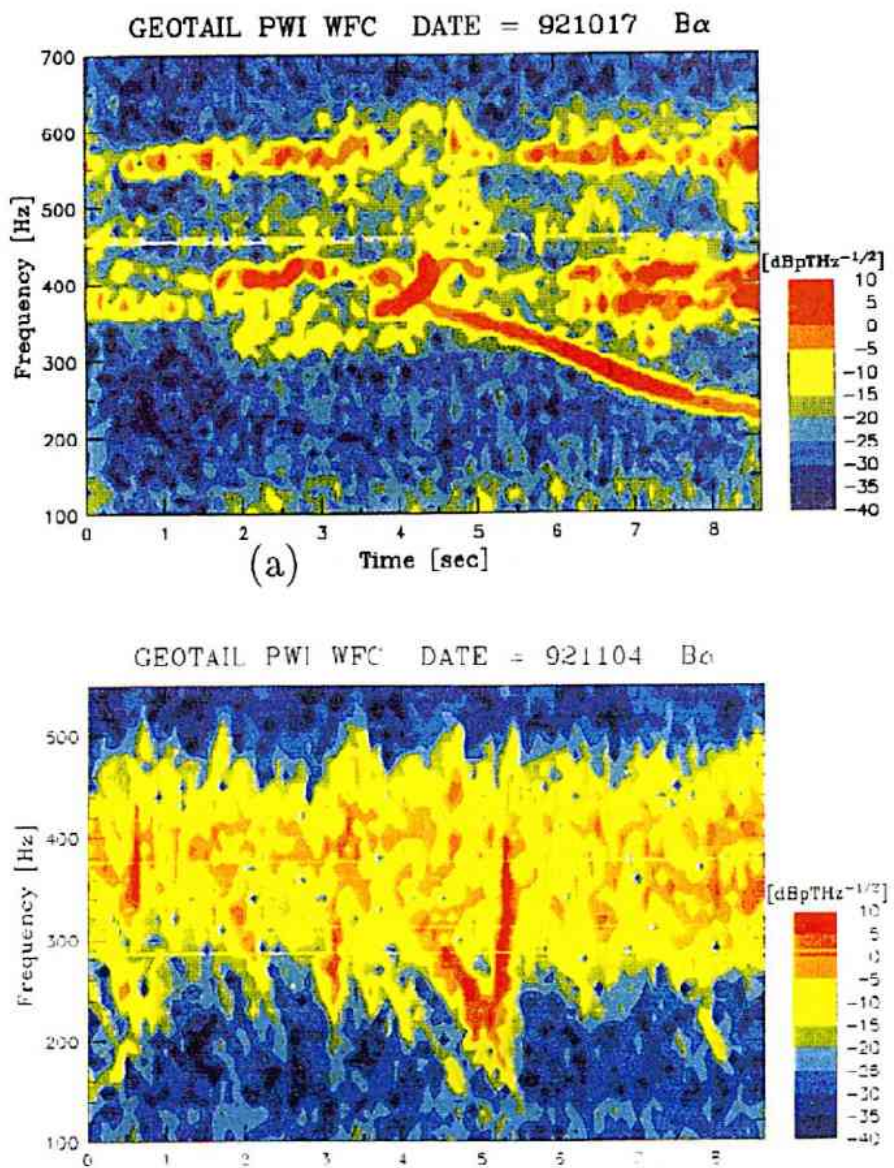

(b) Time [sec]

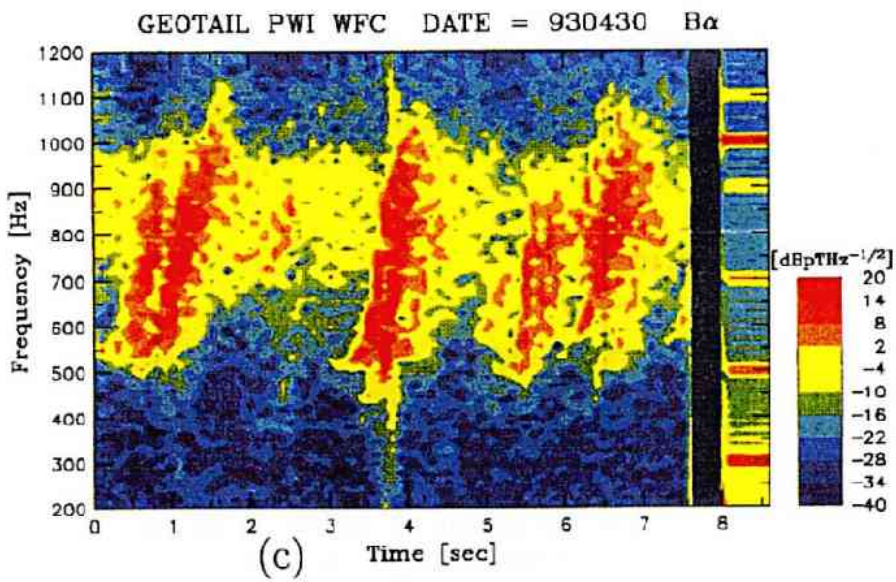

Fig. 2. Spectrograms for (a): rising tones and a falling tone observed at 22:05:02 UT on October 17, 1992, (b): hooks observed at 23:39:59 UT on November 4, 1992, and (c): rising tones at 00:08:27 UT on April 30, 1993. 


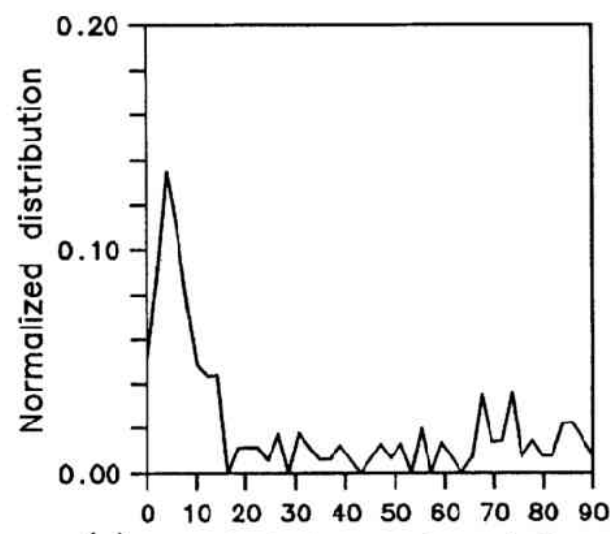

(a) Angle between $k$ and $B_{0}$

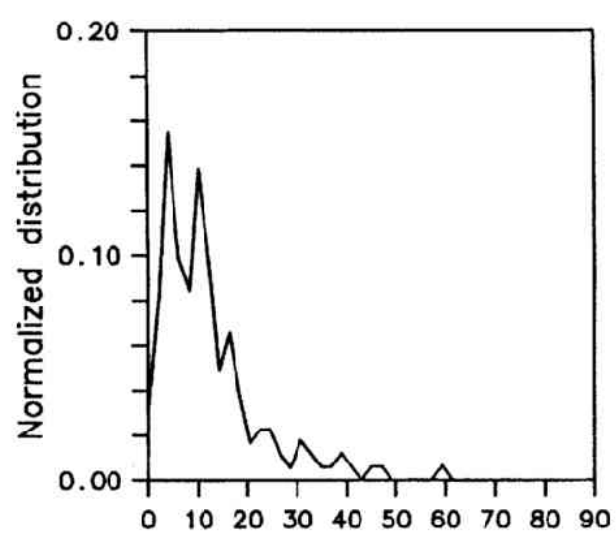

(b) Angle between $k$ and $\mathrm{B}_{0}$

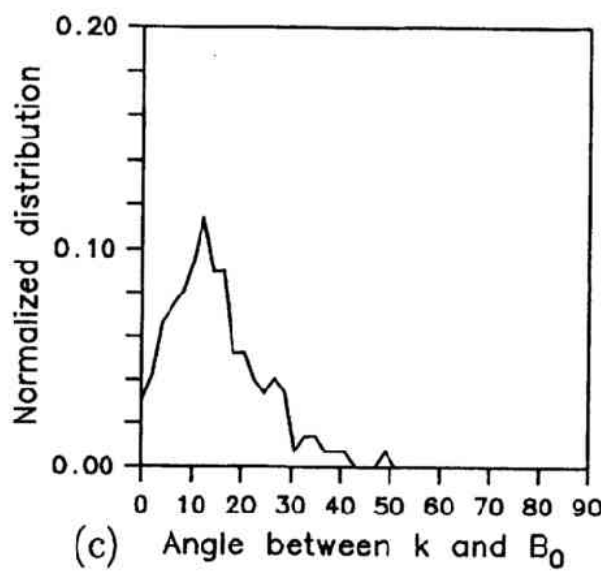

Fig. 3. Wave normal distributions of the chorus emissions with respect to the geomagnetic field line. Figures (a), (b), and (c) correspond to Figs. 2(a), (b) and (c), respectively. 
Figure 3(a) shows the distribution of wave normal angle with respect to the geomagnetic field derived for the falling tone shown in Fig. 2(a). The vertical axis shows the normalized occurrence of the wave normal angles. The confinement of the wave normal angles within 15 degrees indicates that the source location of this emission was relatively nearby if it had propagated in a non-ducted mode (Burton and Holzer, 1974). Though not shown here, the wave normal directions for the rising tones and the falling tone in Fig. 2 are almost parallel and anti-parallel to the geomagnetic field, respectively, indicating that they are generated in different source regions.

Figure 3(b) shows the distribution of the wave normal angle of one of the hooks in Fig. 2(b), where the wave normal of the hook distributes from 5 degrees up to 20 degrees. Angular distribution of the wave normal shown in Fig. 3(c) is for the rising tones in Fig. 2(c). The wave normal in this case distributes over the range between 0 and 30 degrees off the geomagnetic field line. The wider spread in wave normal angles for these hook and rising tones means that they had propagated along non-ducted paths from farther sources than that for the falling tone in Fig. 2(a).

Estimating with Eq. (4) the $E_{z}$ component not observed by the WFC, we can calculate Poynting flux for the chorus emissions by Eq. (5). As an example, Fig. 4 compares the derived wave normal and Poynting
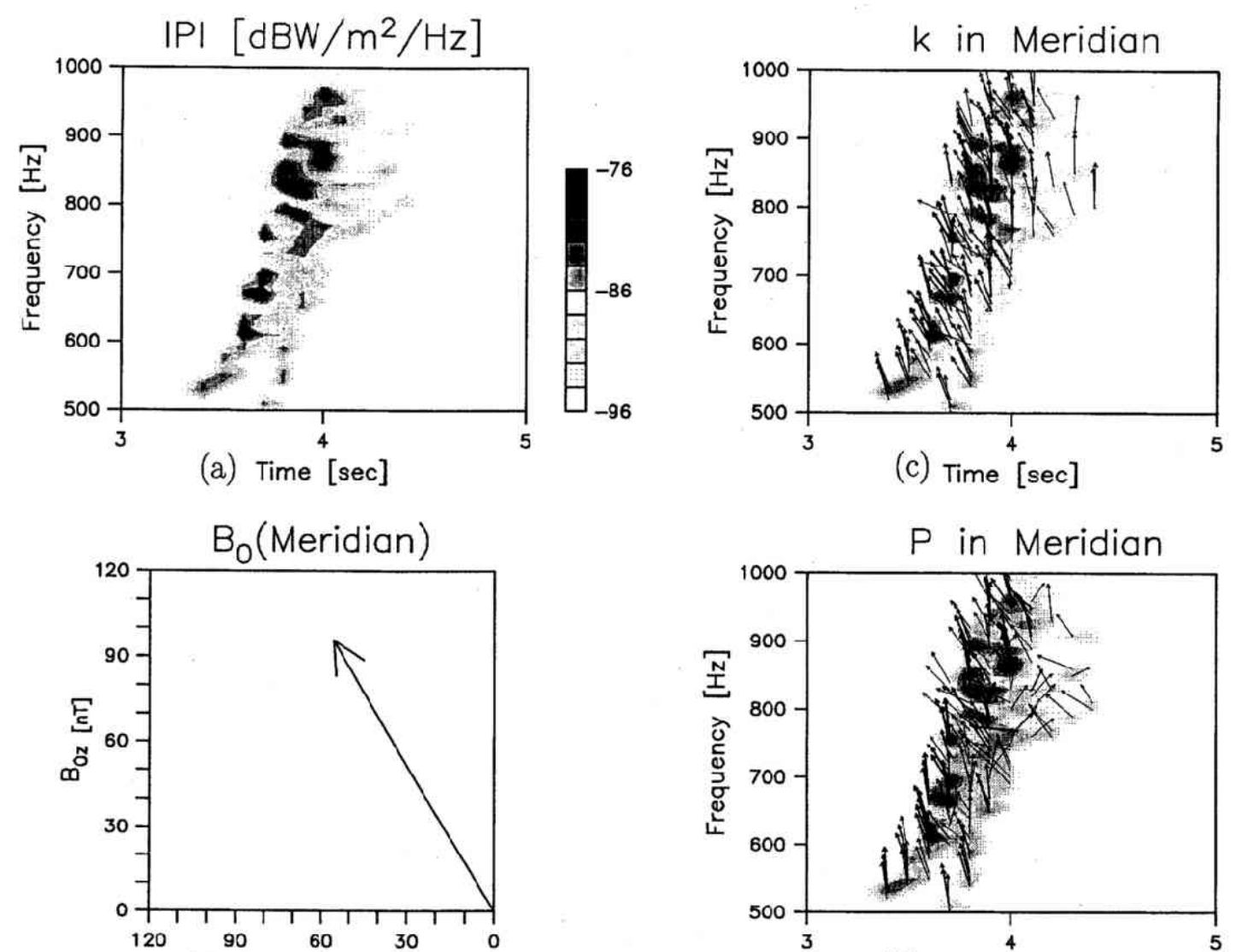

(b) B BGSE-XY $[\mathrm{nT}]$

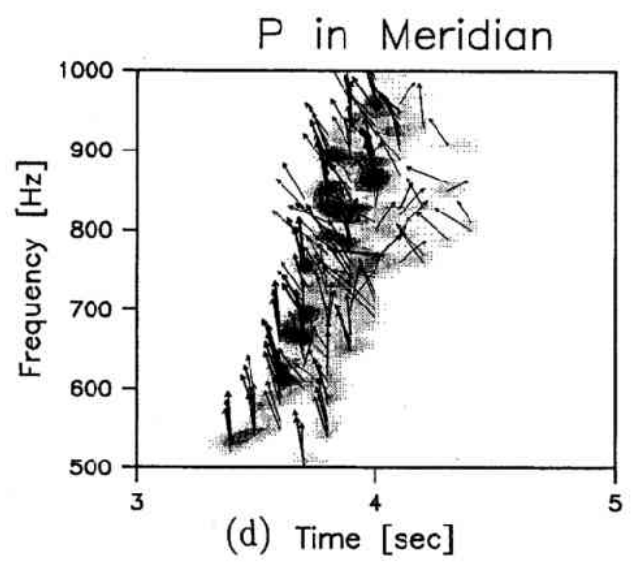

Fig. 4. Wave normal and Poynting directions of one of the rising tones in Fig. 2(c). (a): Spectrogram of Poynting flux. (b): Geomagnetic field line projected onto the meridian plane at the time of observation. (c): Wave normal directions in the meridian plane. (d): Poynting directions in the meridian plane. 


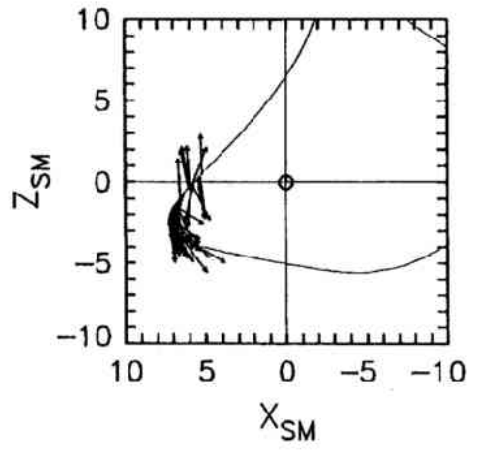

Fig. 5. Wave normal directions of the chorus emissions along the GEOTAIL orbit on October 17-18, 1992, in the $x$ - $z$ plane of the SM coordinate system.

directions with the geomagnetic field direction, for one of the rising tones observed in Fig. 2(c). Figure 4(a) plots a frequency-time structure of Poynting flux, $|P|$, for the rising tone. The maximum value of the Poynting flux is about $-75 \mathrm{dBW} / \mathrm{m}^{2} / \mathrm{Hz}$. Figure $4(\mathrm{~b})$ shows the geomagnetic field line projected onto the magnetic meridian plane. The wave normal and Poynting directions projected onto this plane is plotted by arrows in Figs. 4(c) and 4(d), where the thickness of arrows represents the magnitude of the Poynting flux. The thick arrows for both wave normal and Poynting flux are almost parallel to the geomagnetic field, which is consistent with whistler mode propagation of the chorus emissions in the magnetosphere.

Spatial variation of wave normal directions of chorus emissions observed along the dayside magnetopause skimming of GEOTAIL on October 17, 1992, is shown in Fig. 5, where the Solar Magnetosphere (SM) coordinate system is adopted so that the $x-y$ plane becomes the magnetic equatorial plane. The figure shows the orbit projection onto the $x-z$ plane by the solid line, on which the arrows represent wave normal directions of the chorus emissions observed by the WFC at several locations. The wave normal directions are almost directed northward and southward in the north and south sides of the magnetic equatorial plane, respectively. This clearly suggests that the emissions observed by GEOTAIL along this path were ones generated near the magnetic equatorial plane, which is consistent with the chorus generation mechanism via cyclotron resonance with energetic electrons (Helliwell, 1967).

\section{Summary}

Geotail has observed several types of the chorus emissions in the orbits which skimmed the dayside magnetopause. For each type of the emissions we have analyzed the propagation characteristics by using the five electromagnetic components of wave forms acquired by the WFC. Wave normal and Poynting directions derived for the chorus emissions are consistent with previously proposed generation and propagation mechanisms of chorus emissions.

This kind of analysis makes it possible to investigate a detailed frequency-time structure and propagation characteristics for each element of the emissions. In particular, information on magnitude and direction of Poynting flux as well as those of the wave normal vector will clarify not only source locations of the emissions but also how much energy is transferred from cyclotron resonant electrons into waves during generation of the emissions.

In the future, to investigate generation mechanisms of the chorus emissions observed by GEOTAIL, we will do a statistical analysis of their propagation characteristics, with reference to particle data observed by the onboard particle instruments, the Low Energy Particle (LEP) (Mukai et al., 1994), the Comprehensive Particle Instrument (CPI) (Frank et al., 1994), and the Energetic Particles and Ion Composition (EPIC) (Williams et al., 1994) instruments, from a viewpoint of nonlinear processes involved in the generation mechanisms (Omura et al., 1991). 
The authors thank Profs. A. Nishida, T. Uesugi, and T. Mukai of ISAS for the successful launch of the GEOTAIL spacecraft and maneuvering it into the tail orbit. We are also indebted to Prof. S. Kokubun and his MGF group for the permission to use their magnetic field data. Thanks are also due to other members of PWI team and the graduate students, H. Murata and T. Shiozaki in Kanazawa University.

\section{REFERENCES}

Burton, R. K. and R. E. Holzer, The origin and propagation of chorus in the outer magnetosphere, J. Geophys. Res., 79, 1014 $1023,1974$.

Cornilleau-Wehrlin, J. Etcheto, and R. K. Burton, Detailed analysis of magnetospheric ELF chorus: preliminary results, J. Atmos. Terr. Phys., 38, 1201-1210, 1976.

Frank, L. A., K. L. Ackerson, W. R. Paterson, J. A. Lee, M. R. English, and G. L. Pickett, The Comprehensive Plasma Instrumentation (CPI) for the GEOTAIL spacecraft, J. Geomag. Geoelectr., 46, 23-37, 1994.

Goldstein, B. E. and B. T. Tsurutani, Wave normal directions of chorus near the equatorial source region, J. Geophys. Res., 89, $2789-2810,1984$.

Hayakawa, M., Y. Yamanaka, M. Parrot, and F. Lefeuvre, The wave normals of magnetospheric chorus emissions observed on board GEOS 2, J. Geophys. Res., 89, 2811-2821, 1984.

Helliwell, R. A., A theory of discrete VLF emissions from magnetosphere, J. Geophys. Res., 19, 4773-4790, 1967.

Kokubun, S., T. Yamamoto, M. H. Acuña, K. Hayashi, K. Shiokawa, and H. Kawano, The GEOTAIL magnetic field experiment, J. Geomag. Geoelectr., 46, 7-21, 1994.

Matsumoto, H., I. Nagano, R. R. Anderson, H. Kojima, K. Hashimoto, M. Tsutsui, T. Okada, I. Kimura, Y. Omura, and M. Okada, Plasma wave observations with GEOTAIL spacecraft, J. Geomag. Geoelectr., 46, 59-95, 1994.

Mcans, J. D., Use of the Three-dimensional covariance matrix in analyzing the properties of plasma waves, J. Geophys. Res., 77, $5551-5559,1972$.

Mukai, T., S. Machida, Y. Saito, M. Hirahara, T. Terasawa, N. Kaya, T. Obara, M. Ejiri, and A. Nishida, The Low Energy Particle (LEP) experiment onboard the GEOTAIL satellite, J. Geomag. Geoelectr., 46, 669-692, 1994.

Omura, Y., D. Nunn, H. Matsumoto, and M. J. Rycroft, A review of observational, theoretical and numerical studies of VLF triggered emissions, J. Atmos. Terr. Phys., 53, 351-368, 1991.

Parrot, M. and C. A. Gaye, A statistical survey of ELF waves in a geostationary orbit, Geophys. Res. Lett., 21, 2463-2466, 1994.

Sazhin, S. S. and M. Hayakawa, Magnetospheric chorus emissions: a review, Planet. Space Sci., 40, 681-697, 1992.

Tsurutani, B. T. and E. J. Smith, Two types of magnetospheric ELF chorus and their substorm dependences, J. Geophys. Res., 82, 5112-5128, 1977.

Tsutsui, M., I. Nagano, H. Kojima, K. Hashimoto, H. Matsumoto, and S. Yagitani, Measurements and analysis of antenna admittance aboard the GEOTAIL spacecraft, Radio Sci, 1996 (to be submitted).

Williams, D. J., R. W. McEntire, C. Schlemm, II, A. T. Y. Lui, G. Gloeckler, S. P. Christon, and F. Gliem, GEOTAIL Energetic Particles and Ion Composition Instrument, J. Geomag. Geoelectr., 46, 39-57, 1994. 